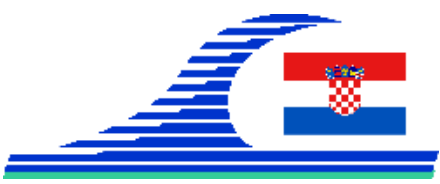

\title{
Nouvelles techniques de déshydratation mécanisée en continu des sédiments. La presse à boues $\mathrm{KDS}^{\circledR}$.
}

\author{
Beatriz BOULLOSA ALLARIZ ${ }^{1}$, Daniel LEVACHER ${ }^{1}$, François THERY ${ }^{2}$
}

1. Université de Normandie, Unicaen, M2C UMR6143 CNRS, 24 rue des Tilleuls, 14000 Caen, France.

beatriz.boullosa-allariz@unicaen.fr ; daniel.levacher@unicaen.fr

2. EDF, R\&D Eco-efficacité des Procédés Industriels, Ecuelles 77818 Moret-sur-Loing cedex, France.

francois.thery@edf.fr

\section{Résumé :}

Les opérations de dragage génèrent une importante quantité de sédiments qui deviennent des déchets dans le cas d'une gestion en terre. Diverses recherches prouvent la possibilité de réutilisation des sédiments non pollués dans le domaine du génie civil. La plupart des applications de réutilisation des sédiments impliquent de déshydrater ces sédiments avant leur valorisation. La déshydratation mécanisée permet de réduire la teneur en eau des sédiments grâce à la combinaison de forces mécaniques. Le paramètre le plus important de ce type de déshydratation est le système de travail. Le système de travail continu permet de suivre une méthodologie de déshydratation 24h/24h. Ce système est utilisé avec la presse à boues Volute $^{\circledR}$ et sa version améliorée KDS ${ }^{\circledR}$. Cette dernière est une presse à boues composée par une succession de disques elliptiques et une plaque de pression située à la fin du cycle de déshydratation. Elle permet la déshydratation continue de boues grâce à la combinaison de la (i) force de cisaillement existant entre les disques et la boue, (ii) la force de compression appliquée par la plaque de pression, (iii) la gravité et (iv) la filtration entre les disques elliptiques et les barres horizontales oscillantes pour déshydrater la boue d'une manière continue. La description de cette machine ainsi que ses paramètres fondamentaux et les premiers résultats obtenus sont présentés dans le présent article.

\section{Mots-clés :}

Déshydratation mécanisée, Système de travail continu, Sédiments, Volute ${ }^{\circledR}$, Presse à boues $\mathrm{KDS}^{\circledR}$.

\section{Introduction}

Le dragage est une opération essentielle pour le développement, l'économie et l'environnement d'une région et/ou pays. Ces opérations génèrent une importante quantité de sédiments de dragage qui deviennent des déchets dans le cas d'une gestion 
Mediterranean rocky coasts:

Features, processes, evolution and problems

en terre. En 2011, un total de 262739 tonnes de sédiments secs a été déposé à terre en France métropolitaine et territoires d'outre-mer (EL FADILI \& MESSAGER, 2015).

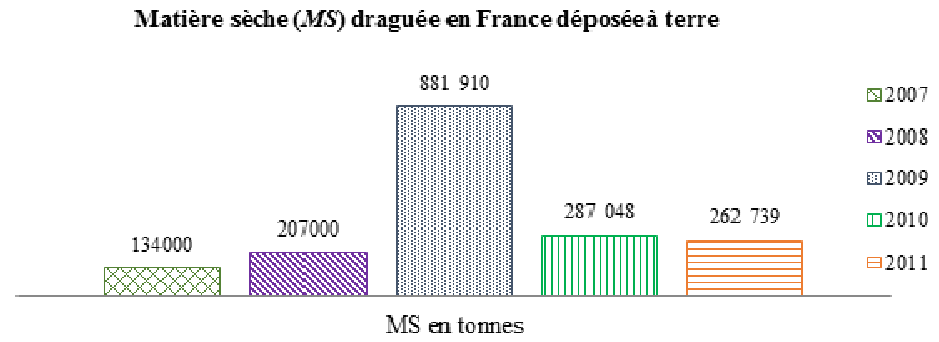

Figure 1. Évolution des tonnages de sédiments dragués déposés à terre en France, de 2007 à 2011.

Diverses recherches ont montré la possibilité de réutilisation des sédiments non pollués dans le domaine du génie civil. L’élaboration de matériaux de construction ou routiers, le remplissage en remblais et/ou carrières, le rechargement de plages et dunes, ou la réalisation d'ouvrages de protection ou digues, sont quelques exemples de valorisation des sédiments dragués et/ou de barrage. La plupart de ces applications impliquent de déshydrater ces sédiments avant de leur valorisation. La déshydratation mécanique mécanisée permet réduire la teneur en eau des sédiments grâce à la combinaison de forces mécaniques. Le paramètre le plus important de ce type de déshydratation est le système ou procédé de travail. Deux systèmes de travail peuvent être différenciés :

a) Un système de travail conventionnel, ou système de travail discontinu, où la machine doit être arrêtée après chaque cycle de déshydratation.

b) Un système de travail en continu qui permet de proposer une méthodologie de déshydratation $24 \mathrm{~h} / 24 \mathrm{~h}$.

Cette communication porte sur la déshydratation mécanisée des sédiments en utilisant une presse à boues $\mathrm{KDS}^{\circledR}$ travaillant en continu.

\section{Presse à boues KDS $^{\circledR}$}

\subsection{Description de la machine}

La presse à boues $\mathrm{KDS}^{\circledR}$ est une machine de déshydratation composée par une succession de disques elliptiques et une plaque de pression située à la fin du cycle d'égouttage. Elle permet la déshydratation continue de boues grâce au (i) cisaillement existant entre les disques et la boue, (ii) la force de compression appliquée par la plaque de pression, (iii) la gravité et (iv) la filtration entre les disques elliptiques et les barres horizontales oscillantes. Quant aux applications, cette presse à boues a la possibilité de déshydrater une large gamme de boues, notamment elle peut être utilisée pour le traitement de déchets alimentaires, de la pêche, agro-industriels, lisiers d'animaux en obtenant des teneurs en eau finales $\mathrm{w}_{\mathrm{f}}$ de $2 \%$ et $6 \%$ (AMCON, 2017 ; ATLANTIQUE 
Mediterranean rocky coasts:

Features, processes, evolution and problems

INDUSTRIE, 2017). Elle n'est pas destinée a priori à des boues minérales ou finement sableuses. L'application aux sédiments fait l'objet de travaux et d'essais spécifiques sur cette machine (BOULLOSA ALLARIZ, 2017), ainsi que l'élaboration d'une méthode de travail afin d'obtenir des $\mathrm{w}_{\mathrm{f}}$ des sédiments d'environ $20 \%-30 \%$ et des concentrations en matières en suspension MES faibles.

\subsection{Principe de fonctionnement}

La presse à boues $\mathrm{KDS}^{\circledR}$ suit le même principe de fonctionnement que la presse à boues Volute $^{\circledR}$. La mixture composée du matériau à déshydrater et de polymères floculants est introduite dans le réservoir de la machine. Ensuite, elle est transportée par un ensemble de disques elliptiques où une force de cisaillement existant entre les disques et la boue permet de réduire considérablement la teneur en eau, voir figures 2.a et 2.b. Une force de compression peut être appliquée à la fin du cycle d'égouttage, voir figure 2.c. Le matériau déshydraté sort après cette plaque de compression, il peut être récupéré à l'aide d'un réservoir pour une future valorisation. En même temps, l'eau s'écoule par les espaces existants entre les disques et grâce aux phénomènes de filtration et de gravité. Elle est stockée dans le collecteur d'eau se trouvant sous la machine. Les eaux résiduaires peuvent être réutilisées ultérieurement. Les dimensions liées à l'encombrement de la machine expérimentale sont données dans la figure 2 .
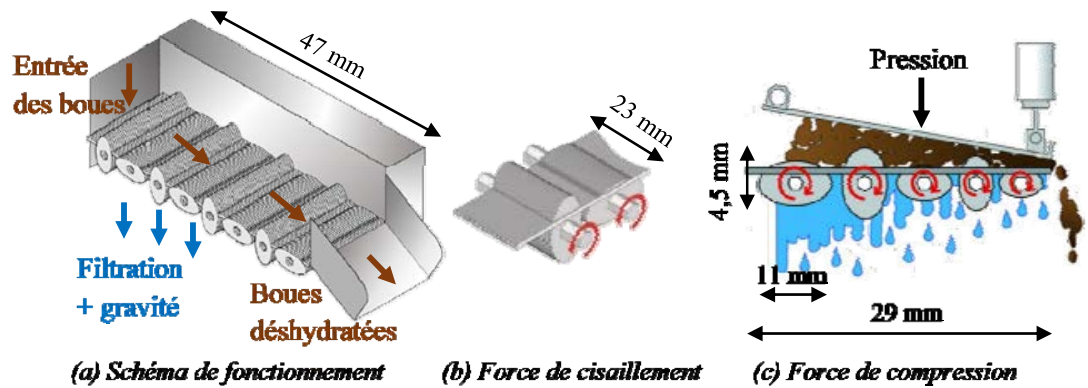

Figure 2. Principe de fonctionnement de la presse $K S^{\circledR}$, (BENENV \& CO, 2017).

\subsection{Paramètres fondamentaux}

Les paramètres-machine qui interviennent dans la presse à boues $\operatorname{KDS}^{\circledR}$ lors de la déshydratation sont : (i) le débit d'entrée du matériau à déshydrater, (ii) la vitesse de rotation des disques elliptiques, (iii) et la pression exercée par le vérin sur la plaque d'appui. Les paramètres d'utilisation retenus pendant les essais sont : le débit d'entrée de $0,1 \mathrm{l} / \mathrm{s}$ à $0,5 \mathrm{l} / \mathrm{s}$, la vitesse de rotation de $2,90 \mathrm{tr} / \mathrm{min}$ à 7,04 tr/min, la pression exercée par le vérin réglable jusqu'à $600 \mathrm{kPa}$ et des teneurs en eau initiales $\mathrm{w}_{0}$ de $400 \%$ et $800 \%$. La $\mathrm{w}_{\mathrm{f}}$ et la MES sont les paramètres mesurés après chaque cycle de déshydratation. 
Mediterranean rocky coasts:

Features, processes, evolution and problems

\section{Premiers résultats dans des ces tests}

Des boues de forage en provenance de Chine et de kaolinite Speswhite ont été déshydratées. Pour ces premiers essais on constate que la machine $\mathrm{KDS}^{\circledR}$ fournit un coefficient de réduction de déshydratation $\left(\mathrm{w}_{0} / \mathrm{w}_{\mathrm{f}}\right)$ plus élevé quand le matériau à déshydrater est composé principalement d'argiles. Pour la kaolinite, la machine KDS ${ }^{\circledR}$ réduit à la moitié sa quantité d'eau. Le choix du floculant reste primordial, efficacité et quantité doivent être considérées tout en sachant qu'un dosage en floculant le plus réduit diminue le cout de déshydratation.

Tableau 1. Caractéristiques des sédiments après déshydratation avec la machine $K_{D S}{ }^{\circledR}$.

\begin{tabular}{ccccccc}
\hline $\begin{array}{c}\text { Sédiments } \\
\text { (origine) }\end{array}$ & $\begin{array}{c}\text { Volume initial de } \\
\text { sédiments }\end{array}$ & $\begin{array}{c}\text { Quantité de } \\
\text { floculant }\end{array}$ & Floculant & wo $_{\mathbf{0}}$ (\%) & $\boldsymbol{w}_{\boldsymbol{f}}$ (\%) & $\begin{array}{c}\text { Facteur de } \\
\text { réduction }\end{array}$ \\
\hline Chine & $3 \mathrm{l}$ & $70 \mathrm{ml}$ & FA 920 VHR & 183,49 & 126,35 & 1,45 \\
Chine & $6 l$ & $300 \mathrm{ml}$ & AH 912 SH & 183,49 & 158,24 & 1,16 \\
Chine & $6 l$ & $360 \mathrm{ml}$ & AN 923 SH & 183,49 & 171,38 & 1,07 \\
Kaolin & $2 l$ & $85 \mathrm{ml}$ & AN 923 & 275,00 & 145,01 & 1,90 \\
\hline
\end{tabular}

Note : La préparation du floculant a été réalisée avec une concentration de $1 \mathrm{~g} / \mathrm{l}$ pour tous les sédiments sauf pour le kaolin qui a été préparé avec concentration de $10 \mathrm{~g} / \mathrm{l}$.

\section{Conclusion}

La déshydratation mécanique présente une solution efficace, rapide et peu dépendante des caractéristiques physico-chimiques des sédiments et de la météo locale dans le cas d'un séchage naturel. Les nouveaux systèmes de déshydratation mécanique proposent un système de travail continu. Pour des raisons de conception, la presse à boue Volute ${ }^{\circledR}$ et sa version améliorée $\operatorname{KDS}^{\circledR}$ n'ont jamais été testées avec des sédiments La prise en main de la presse à boues $\mathrm{KDS}^{\circledR}$ a permis d'établir des hypothèses de fonctionnement et la connaissance des paramètres de fonctionnement pour la déshydratation des sédiments.

\section{Références bibliographiques}

AMCON. (2017). Amenity Convenience. http://en.amcon.co.jp

ATLANTIQUE INDUSTRIE. (2017). http://atlantiqueindustrie.fr//

BENENV \& CO. (2017). Benenfit the Environment. http://en.benenv.com/

BOULLOSA ALLARIZ B. (2017). Étude de la déshydratation mécanisée Volute ${ }^{\circledR}$ $K_{D S}{ }^{\circledR}$. Principe de fonctionnement et paramètres étudiés. Rapport M2C-COVASEDEDF-04, Université de Caen, Caen, France, 38 p.

EL FADILI M., MESSAGER M. (2015). Enquête dragage 2011. Synthèse des données. CEREMA Editeur, France, 39p. 